\title{
A novel missense variant in the DIAPH1 gene in a Korean family with autosomal dominant nonsyndromic hearing loss
}

\author{
Tae-Hun Kang ${ }^{1,2 \ddagger}$, Jeong-In Baek ${ }^{3 \ddagger}$, Borum Sagong ${ }^{1}$, Hong-Joon Park ${ }^{4}$, \\ Chan Ik Park ${ }^{3}$, Kyu-Yup Lee ${ }^{5 *}$ and Un-Kyung Kim ${ }^{1,2 \dagger}$ \\ ${ }^{1}$ Department of Biology, College of Natural Sciences, Kyungpook National University, 80 Daehak-ro, Buk-gu, \\ Daegu 41566, Republic of Korea \\ ${ }^{2}$ School of Life Sciences, BK21 Plus KNU Creative BioResearch Group, Kyungpook National University, \\ 80 Daehak-ro, Buk-gu, Daegu 41566, Republic of Korea \\ ${ }^{3}$ Department of Aroma Applied Industry, College of Herbal Bio-industry, Daegu Haany University, \\ 1 Hanuidae-ro, Kyungsan, Gyeongsangbuk-do 38610, Republic of Korea \\ ${ }^{4}$ Soree Ear Clinics, 435 Hakdong-ro, Gangnam-gu, Seoul 06068, Republic of Korea \\ ${ }^{5}$ Department of Otorhinolaryngology-Head and Neck Surgery, School of Medicine, \\ Kyungpook National University, 130 Dongdeok-ro, Jung-gu, \\ Daegu 41944, Republic of Korea
}

(Received 19 July 2016, accepted 19 October 2016; J-STAGE Advance published date: 21 December 2016)

Hair cells in the cochlea display highly regulated actin polymerization, which is mediated by the human diaphanous-related formin 1 gene (DIAPH1; also called DFNA1, DIA1). DFNA1, the first type of autosomal dominant nonsyndromic hearing loss (ADNSHL), is known to be associated with mutations in DIAPH1. However, no genetic study of DFNA1 in Koreans with hearing loss has yet been reported. A 51-year-old patient in a Korean family with ADNSHL was examined by pure-tone audiometry, and genetic analysis of DIAPH1 was performed. A novel variant, p.I530S (c.1589T > G), was identified in the DIAPH1 gene, and the mutation was located in the highly conserved coiled-coil domain of the DIA1 protein, where an amino acid substitution was predicted to change the domain structure. Further functional investigations will provide more information to help us understand the role of DIAPH1 in maintenance of hair cell function in the auditory pathway.

Key words: $D I A P H 1$, diaphanous-related formin 1, DFNA1, autosomal dominant nonsyndromic hearing loss in Koreans

Hair cells in the inner ear are mechanoreceptors that mediate mechanoelectrical transduction in the auditory pathway (Frolenkov et al., 2004). Stereocilia, which are apical modifications of the hair cells in the cochlea, are the key organelles that convert the mechanical stimulation of sound waves into electrical signals in the hair cells (Hudspeth and Corey, 1977). The stereocilium is composed of actin filaments that are tightly regulated by polymerization and depolymerization to maintain the structure of stereocilia (Tilney et al., 1983; Breneman et al., 2009). Actin polymerization is mediated by the direct interaction between formin proteins and profilinactin complexes (Tilney et al., 1983; Nouvian et al., 2006;

\footnotetext{
Edited by Rimantas Kodzius

* Corresponding author. E-mail: kylee@knu.ac.kr

$\uparrow$ Corresponding author. E-mail: kimuk@knu.ac.kr

$\ddagger$ These authors contributed equally to this work.

DOI: http://doi.org/10.1266/ggs.16-00041
}

Breneman et al., 2009). Formins recruit these complexes to the barbed ends of the growing actin filaments, leading to the assembly of new actin polymers (Krebs et al., 2001; Evangelista et al., 2003; Suarez et al., 2015). Actin polymerization is involved in various central cellular processes such as differentiation, migration, adhesion, signaling and gene expression, as well as structural maintenance (Mattila and Lappalainen, 2008).

The diaphanous-related formins (DRFs), members of the formin family, directly promote actin filament growth (Wallar and Alberts, 2003). The human diaphanousrelated formin 1 (DIA1) protein is encoded by the DIAPH1 gene (Lynch et al., 1997). DIAPH1 is located on chromosome 5q31 and consists of 28 exons, and is known to be associated with autosomal dominant nonsyndromic hearing loss (ADNSHL) (Lynch et al., 1997). To date, only a small number of mutations in this gene have been reported as the cause of ADNSHL, including the Costa Rican kindred with low-frequency progressive hearing 
loss (Lynch et al., 1997; Baek et al., 2012). In this study, we report a novel missense variant in the DIAPH1 gene in a Korean patient with ADNSHL.

A 51-year-old male with sensorineural hearing loss (HL) presented to our hospital. He had been diagnosed with HL a decade previously. This patient (III-1) had a family history of HL (Fig. 1A), the heredity pattern of which was autosomal dominant. The threshold hearing levels of the patient were examined by pure-tone audiometry in a sound-proof room, at sound frequencies of 250, 500, 1,000, 2,000, 4,000 and 8,000 Hz. The level of HL was based on the standards of a previous study (Sagong et al., 2016). The pure-tone audiogram of the patient revealed a steeply sloping form (Fig. 1B).

For genetic analysis, 100 unrelated individuals with normal hearing were recruited from Kyungpook National University as control subjects. The participants pro- vided written informed consent before the study according to the protocol approved by the Ethics Committee of Kyungpook National University Hospital. Genomic DNA from blood samples of the patient and control individuals was extracted using the FlexiGene DNA Extraction Kit (Qiagen, Hilden, Germany). Primers were designed using 3web (http://primer3.ut.ee) to amplify each coding region containing flanking splice junctions of DIAPH1 (Supplementary Table S1). Amplification of each exon was carried out by PCR using $h$-Taq DNA polymerase (Solgent, Daejeon, Korea), and the PCR products were confirmed on a $1.5 \%$ agarose gel by electrophoresis. Exonuclease I (USB, Cleveland, OH, USA) and shrimp alkaline phosphatase (USB) were used to purify the PCR products, and their sequences were determined by thermal cycling PCR using the BigDye Terminator v3.1 Cycle Sequencing Kit (Applied Biosystems, Foster City, CA, USA). Sanger
(A)

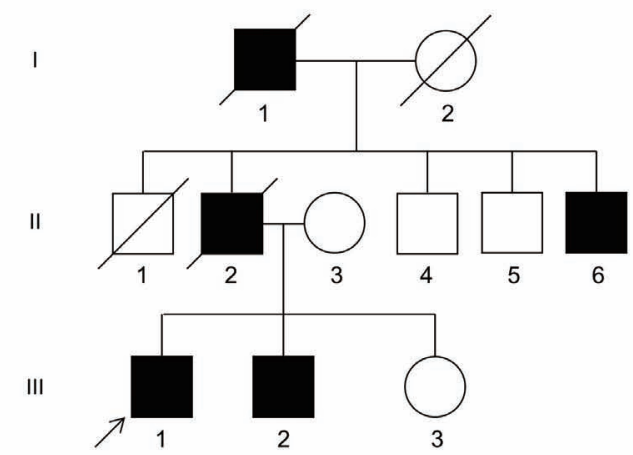

(C)

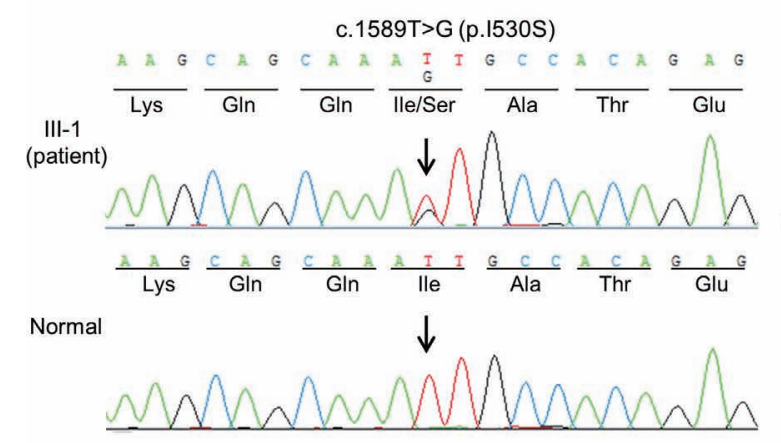

(B)

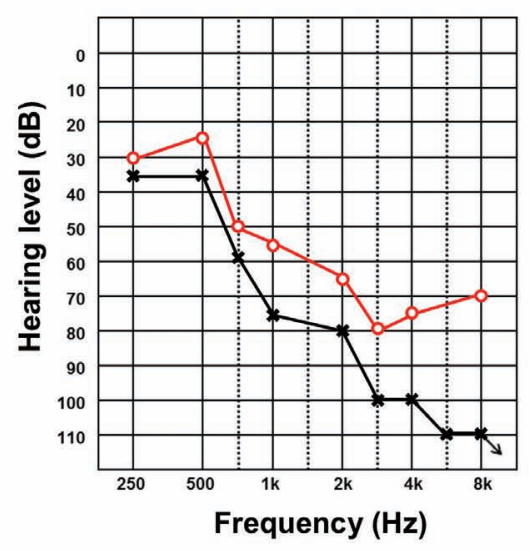

(D)

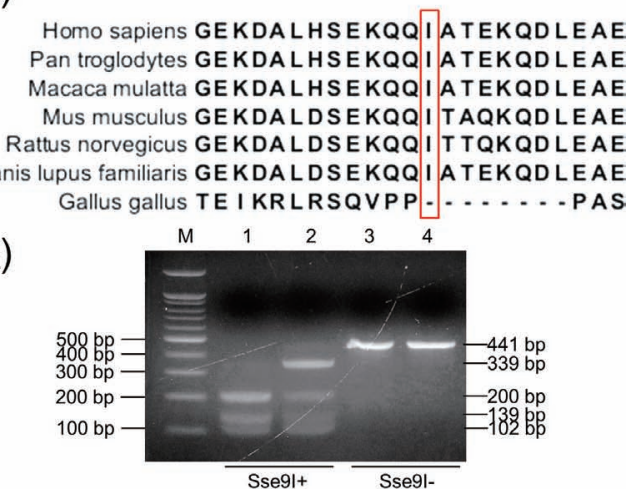

Fig. 1. The p.I530S variant in the DIAPH1 gene in family SR11-1750. A. Pedigree diagram of family SR11-1750. Filled and open symbols indicate affected and unaffected individuals, respectively. The arrow indicates the proband of the family. Slashes indicate deceased individuals. B. Pure-tone audiogram for left and right ears of the proband. Air conduction threshold measured by pure-tone audiometry of the proband. Circles and crosses represent the thresholds of the right and left ear, respectively. The arrow indicates no response at hearing levels above $110 \mathrm{~dB}$. C. Chromatogram of nucleotide sequences showing the c.1589T > G variant (p.I530S). Arrows represent the c.1589T > G variant site. D. Evolutionary conservation of DIA1 amino acid sequences around I530 (boxed) in various species. E. PCR amplification products of exon 15 of the DIAPH1 gene after digestion with Sse9I. This enzyme cuts DNA sequences at N/AATTN, and was used to confirm the c.1589T > G variant. Lane M, 100-bp molecular size marker; lane 1, normal control with enzyme digestion; lane 2, heterozygote for c.1589T > G with enzyme digestion; lane 3, normal control without enzyme digestion; lane 4, heterozygote for c.1589T > G without enzyme digestion. In the normal control, digestion yielded three fragments of 102 , 139 and 200 bp (lane 1). In the heterozygote for c.1589T > G, digestion yielded four fragments of 102, 139, 200 and 339 bp (lane 2). The 339-bp band was not present in the digested normal allele. 


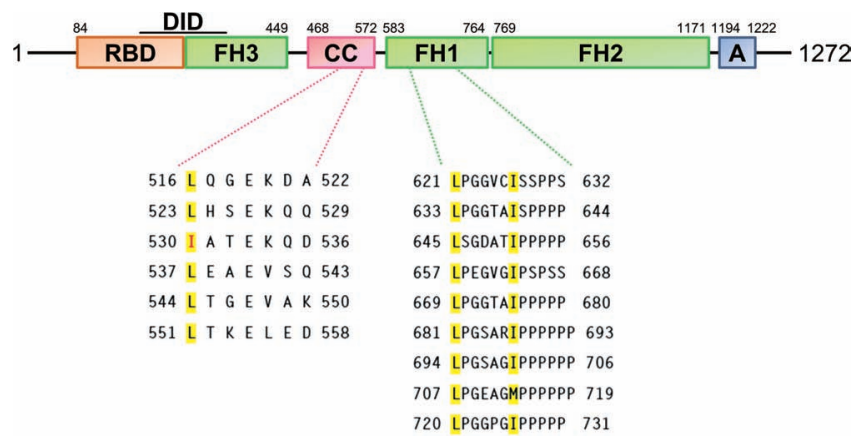

Fig. 2. Domains of DIA1. Abbreviations: RBD, Rho-GTPase binding domain; FH3, formin homology 3 domain; DID, diaphanous inhibitory domain; CC, coiled-coil domain; FH1, formin homology 1 domain; FH2, formin homology 2 domain; A, diaphanous autoinhibitory domain. The repeating patterns of amino acids in the $\mathrm{CC}$ and $\mathrm{FH} 1$ domains were sequenced. The nonpolar residue is colored in yellow boxes. Amino acid 530 (isoleucine) is highlighted in red.

sequencing was carried out by capillary electrophoresis on a 3130xl Genetic Analyzer (Applied Biosystems). For sequence data analysis, Sequencing Analysis v5.2 (Applied Biosystems), SeqScape v2.5 (Applied Biosystems) and ChromasPro v1.7 (Technelysium, Tewantin, QLD, Australia) were used. Finally, the sequence of each exon was compared with that of the reference DIAPH1 (NG_011594.1, NM_005219.4) sequence registered in the NCBI (http://www.ncbi.nlm.nih.gov/) database. To investigate the probable pathogenicity and novelty of detected variants, several different exome databases were used as references: the 1000 Genomes Project Browser (http:// www.1000genomes.org/), dbSNP (http://www.ncbi.nlm.nih. gov/snp/), the Human Genetic Variation database (http:// www.genome.med.kyoto-u.ac.jp/), the NHLBI GO Exome Sequencing Project (http://evs.gs.washington.edu/EVS/) and the Exome Aggregation Consortium (ExAC) browser (http://exac.broadinstitute.org/). Conservation of the DIA1 protein sequence among species was compared using CLC Sequence Viewer v6.0 (CLC Bio, Aarhus, Denmark), and the change of structural stability caused by mutations was predicted using MUpro, a web-based program to predict how single-site amino acid mutation affects protein stability (http://www.ics.uci.edu/ baldig/mutation.html). The restriction enzyme Sse9I (Enzynomics, Daejeon, Korea) was used to digest the PCR products for the 100 control subjects.

Genetic analysis of the DIAPH1 gene revealed that the patient had a nonsynonymous variant, p.I530S (C.1589T > G), in the heterozygous state. This mutation has not been identified in any exome database including the 1000 Genomes Project Browser and dbSNP databases, suggesting the novelty of the p.I530S variant. It was a single-nucleotide change of thymine to guanine at nucleotide position 1589, which causes an amino acid substitution of isoleucine to serine at amino acid position 530 (Fig. 1C), changing the molecular characteristic of the amino acid from nonpolar to polar. This amino acid change was predicted by MUpro to decrease the structural stability of the protein, with a highly significant confidence score of -1 , suggesting that the p.I530S mutation would destabilize the coiled-coil (CC) domain in the DIA1 protein. Moreover, this variant was located in a region of DIA1 that is highly conserved among various mammalian species (Fig. 1D), suggesting that this region, including isoleucine 530 , is important for protein function. The PCR products amplified from the 100 normal controls were not digested by Sse9I, indicating that these unrelated individuals did not harbor the p.I530S variant (Fig. 1E).

DIAPH1 was reported as the first causative gene of ADNSHL in a large Costa Rican kindred in 1997 (Lynch et al., 1997), but since then no further genetic studies of DIAPH1-related ADNSHL have been published. However, we recently reported a novel and probably pathogenic mutation in DIAPH1 in a patient with ADNSHL, identified by targeted massively parallel sequencing (Baek et al., 2012), which suggested the desirability of investigating the prevalence of DIAPH1-related ADNSHL in the Korean population. In this study, we have now found another novel missense variant in a different patient, suggesting the possibility that DIAPH1 is not a minor causative gene of hearing loss in Koreans. Further genetic analysis of DIAPH1 in a larger patient population will provide useful genetic information about Korean hereditary hearing loss.

Although DIA1-regulated actin polymerization is essential for cell activity (Mattila and Lappalainen, 2008), the pathogenic effect of DIA1 malfunction caused by structural change is not well known in hair cells. The DIA1 protein contains CC, Rho-GTPase binding, diaphanous inhibited, formin homology (FH)1, FH2, FH3, and diaphanous autoinhibitory domains (Zigmond, 2004; Higgs, 2005). FH domains play an important role in actin polymerization by interacting with profilin-actin complexes (Wallar and Alberts, 2003; Higgs, 2005). CCs are also important for protein structural stabilization, regulation of other downstream genes and interaction with other molecules (Mason and Arndt, 2004; Parry et al., 2008). Hydrophobic nonpolar residues such as leucine, isoleucine and valine are key to maintaining the stable helical structure of CCs (Harbury et al., 1993; Parry et al., 2008). This indicates that chemical features of individual amino acids in the CCs can affect or even determine structural stabilization or functions of the protein. The p.I530S variant found in this study is located in a repeated region of nonpolar residues in the $\mathrm{CC}$ domain (Fig. 2). Therefore, the change of nonpolar isoleucine to polar serine in this region might have the potential to affect the original repeating pattern of nonpolar residues, leading to structural alteration of the protein. Since the cells in the inner ear are highly sensitive to actin fibers and actin filament networks, func- 
tional loss of actin regulators such as DIA1 may lead to failure in maintaining the normal morphology and functions of the cells (Tilney et al., 1980). In conclusion, we report a genetic analysis of the DIAPH1 gene in a Korean family with ADNSHL. Further functional investigations should provide stronger evidence to test our hypothesis that the p.I530S variant has the potential to disrupt the maintenance of normal DIA1 structure and functions.

This work was supported by the National Research Foundation (NRF) of Korea (2015R1A2A2A01003438), and by the Bio \& Medical Technology Development Program of the National Research Foundation of Korea, funded by the Ministry of Science, Information and Communications Technology and Future Planning (2014M3A9D5073865).

\section{DECLARATION OF INTEREST}

The authors have no conflict of interest to declare.

\section{REFERENCES}

Baek, J. I., Oh, S. K., Kim, D. B., Choi, S. Y., Kim, U. K., Lee, K. Y., and Lee, S. H. (2012) Targeted massive parallel sequencing: the effective detection of novel causative mutations associated with hearing loss in small families. Orphanet J. Rare Dis. 7, 60 .

Breneman, K. D., Brownell, W. E., and Rabbitt, R. D. (2009) Hair cell bundles: flexoelectric motors of the inner ear. PLoS One 4, e5201.

Evangelista, M., Zigmond, S., and Boone, C. (2003) Formins: signaling effectors for assembly and polarization of actin filaments. J. Cell Sci. 116, 2603-2611.

Frolenkov, G. I., Belyantseva, I. A., Friedman, T. B., and Griffith, A. J. (2004) Genetic insights into the morphogenesis of inner ear hair cells. Nat. Rev. Genet. 5, 489-498.

Harbury, P. B., Zhang, T., Kim, P. S., and Alber, T. (1993) A switch between two-, three-, and four-stranded coiled coils in GCN4 leucine zipper mutants. Science 262, 1401-1407.

Higgs, H. N. (2005) Formin proteins: a domain-based approach. Trends Biochem. Sci. 30, 342-353.

Hudspeth, A. J., and Corey, D. P. (1977) Sensitivity, polarity, and conductance change in the response of vertebrate hair cells to controlled mechanical stimuli. Proc. Natl. Acad. Sci. USA 74, 2407-2411.

Krebs, A., Rothkegel, M., Klar, M., and Jockusch, B. M. (2001) Characterization of functional domains of mDia1, a link between the small GTPase Rho and the actin cytoskeleton. J. Cell Sci. 114, 3663-3672.

Lynch, E. D., Lee, M. K., Morrow, J. E., Welcsh, P. L., Leon, P. E., and King, M. C. (1997) Nonsyndromic deafness DFNA1 associated with mutation of a human homolog of the Drosophila gene diaphanous. Science 278, 1315-1318.

Mason, J. M., and Arndt, K. M. (2004) Coiled coil domains: stability, specificity, and biological implications. ChemBioChem 5, $170-176$

Mattila, P. K., and Lappalainen, P. (2008) Filopodia: molecular architecture and cellular functions. Nat. Rev. Mol. Cell Biol. 9, 446-454.

Nouvian, R., Beutner, D., Parsons, T. D., and Moser, T. (2006) Structure and function of the hair cell ribbon synapse. J. Membr. Biol. 209, 153-165.

Parry, D. A., Fraser, R. D., and Squire, J. M. (2008) Fifty years of coiled-coils and alpha-helical bundles: a close relationship between sequence and structure. J. Struct. Biol. 163, 258-269.

Sagong, B., Baek, J. I., Bok, J., Lee, K. Y., and Kim, U. K. (2016) Identification of a nonsense mutation in the STRC gene in a Korean family with moderate hearing loss. Int. J. Pediatr. Otorhinolaryngol. 80, 78-81.

Suarez, C., Carroll, R. T., Burke, T. A., Christensen, J. R., Bestul, A. J., Sees, J. A., James, M. L., Sirotkin, V., and Kovar, D. R. (2015) Profilin regulates F-actin network homeostasis by favoring formin over Arp2/3 complex. Dev. Cell 32, 43-53.

Tilney, L. G., Derosier, D. J., and Mulroy, M. J. (1980) The organization of actin filaments in the stereocilia of cochlear hair cells. J. Cell Biol. 86, 244-259.

Tilney, L. G., Egelman, E. H., DeRosier, D. J., and Saunder, J. C. (1983) Actin filaments, stereocilia, and hair cells of the bird cochlea. II. Packing of actin filaments in the stereocilia and in the cuticular plate and what happens to the organization when the stereocilia are bent. J. Cell Biol. 96, 822-834.

Wallar, B. J., and Alberts, A. S. (2003) The formins: active scaffolds that remodel the cytoskeleton. Trends Cell Biol. 13, $435-446$.

Zigmond, S. H. (2004) Formin-induced nucleation of actin filaments. Curr. Opin. Cell Biol. 16, 99-105. 